\title{
MicroRNA-130a Targets MAP3K12 to Modulate Diabetic Endothelial Progenitor Cell Function
}

\author{
Meng Ye ${ }^{a}$ Dan Lia Jian Yang ${ }^{b}$ Jing Xie ${ }^{a}$ Fei Yua Yushui Ma ${ }^{a}$ Xuchao Zhu \\ Jinwei Zhaoc Zhongwei Lva \\ aDepartment of Nuclear Medicine, Shanghai $10^{\text {th }}$ People's Hospital, School of Medicine, Tongji \\ University, Shanghai, b Department of Nuclear Medicine, Changhai Hospital, the Second Military \\ Medical University of Chinese PLA, Shanghai, 'Interventional and Vascular Surgery Department, \\ Laiyang Centre Hospital, Yantai City, People's Republic of China
}

\section{Key Words}

MicroRNA-130a • Endothelial progenitor cell • JNK signal pathway • MAP3K12

\begin{abstract}
Aims: The aim of the present study was to explore the influence of microRNA (miR)-130a dysregulation on the JNK signal pathway through its target MAP3K12 in diabetic endothelial progenitor cells (EPCs). Methods: The expression of miR-130a was compared between diabetic and normal EPCs. Computational target prediction was performed to identify MAP3K12 as a functionally relevant target of miR-130a in EPCs. The role of miR-130a was investigated regarding its anti-apoptotic effects and its role on the regulation of EPC function was evaluated through the negative regulation of the JNK signal pathway. Results: MiR-130a expression was significantly downregulated in diabetic EPCs, and cell proliferation was reduced in EPCs under high glucose condition. miR-130a inhibited the JNK pathway by targeting MAP3K12, contributing to its anti-apoptotic effect and the maintenance of EPC function. In diabetic EPCs, high glucose affects the expression of miR-130a, inducing sustained JNK activation and promoting EPC apoptosis and dysfunction. Conclusions: Downregulation of miR-130a may underlie endothelial dysfunction in diabetes through the activation of JNK signal pathway.
\end{abstract}

M. Ye, D. Li and J. Yang are equally contribute to this paper.

Zhongwei Lv,

Jinwei Zhao,

\section{KARGER 125}

Department of Nuclear Medicine, Shanghai 10th People's hospital, 301 Yanchang Road, Shanghai, 200072, (People's Republic of China) and Interventional and Vascular Surgery Department, Laiyang Centre Hospital, Yantai City, Shandong Province, 265200, (People's Republic of China); E-Mail Izwkxy@163.com, E-Mail 7132603@qq.com 


\section{Cellular Physiology Cell Physiol Biochem 2015;36:712-726 \\ \begin{tabular}{l|l} 
and Bioch 10.1159/000430132 & $\begin{array}{l}\text { ○ 2015 S. Karger AG, Basel } \\
\text { www.karger.com/cpb }\end{array}$ \\
\hline
\end{tabular}}

\section{Introduction}

Diabetes mellitus (DM) is associated with various vascular complications and is an important risk factor for cardiovascular diseases. Endothelial dysfunction contributes to the pathogenesis of vascular diseases in diabetes, and endothelial progenitor cell (EPC) plays a critical role in maintaining vascular endothelial function [1]. Bone marrow-derived EPCs migrate to ischemia tissues at the sites of vessel injury and differentiate into mature endothelial cells, where they are involved in the recovery of endothelial function [2]. Hyperglycemia is associated with endothelial dysfunction and is one of the major causes of vascular complications in patients with DM [3]. DM is associated with low levels of circulating EPCs and EPC dysfunction, in particular impaired proliferation, adhesion and migration [4, 5]. Studies have demonstrated that diabetes reduces the number of EPCs and adversely affects the functional capacity of existing EPCs. Therefore, elucidation of the mechanism of DM-induced EPC dysfunction is important to develop effective strategies for the treatment of the complications of DM.

Micro-RNAs (miRNAs) are small non-coding RNAs that specifically bind to the 3 '-untranslated region (UTR) of target cellular mRNA, leading to either mRNA degradation or inhibition of translation [6]. miRNA plays an important role in cell growth, differentiation, and apoptosis. Many miRNAs are involved in the pathogenesis of diabetic complications associated with EPC dysfunction, such as miR-34a, miR-107 and miR-126 [7-9]. We used microarray analysis technology to screen for miRNAs altered in EPCs derived from type II DM patients and identified miR-130a as a downregulated miRMA in EPCs. A previous study suggested that miR-130a regulates vascular repair in DM by targeting RunX3, and that it plays an important role in maintaining endothelial cell proliferation, migration and tubulogenic activity [10]. In the present study, we used the Targetscan Program (http//:www. targetscan. org) and predicted MAP3K12 as a novel target of miR-130a in EPCs.

MAP3K12 is the activator of dual specificity mitogen-activated protein kinase (MAPK) phosphatase MKK7, which is the upstream specific activated enzyme of the c-Jun $\mathrm{NH}_{2}$ terminal kinase (JNK) [11]. As a subgroup of the MAPK signal pathway, the JNK signal pathway is activated primarily by exposure to environmental stresses, such as osmotic pressure and oxidative stress [12]. The JNK signal pathway plays a significant role in various physiological processes such as cell proliferation, differentiation, development, inflammatory response and apoptosis [13]. Recent genetic evidence and emerging pharmacological data indicate that activated JNK may be critical in the pathogenesis of insulin resistance, diabetes and atherosclerosis [14]. Moreover, activation of JNK mediates vascular endothelial cell apoptosis induced by high glucose [5].

The aim of the present study was to examine the role of miR-130a downregulation in EPC dysfunction and the involvement of the JNK signal pathway. The results of this study will advance our understanding of the role of miRNAs in EPC dysfunction in DM.

\section{Materials and Methods}

\section{Patient recruitment}

Patients with Type II DM ( $\mathrm{n}=20)$ were recruited from Shanghai Tenth People's Hospital (Shanghai, China) and $20 \mathrm{~mL}$ of blood samples were collected from peripheral veins before treatment. All patients provided written informed consents. Blood samples were collected from healthy individuals $(n=20)$ who presented to the hospital for preventive check-ups and used as normal controls. All protocols were approved by the Research Ethics Board of Shanghai Tenth People's Hospital. Patients' baseline characteristics were summarized in Table 1.

Isolation and cultivation of EPCS

Peripheral human blood (20 mL) was added in $1 \mathrm{~mL}$ heparin and separated by Ficoll-Isopaque Plus (Histopaque-1077, USA) density gradient centrifugation within $2 \mathrm{~h}$ after collection. CD133 ${ }^{+}$cells were 


\section{Cellular Physiology Cell Physiol Biochem 2015;36:712-726 \begin{tabular}{l|l|l} 
DOI: 10.1159/000430132 & O 2015 S. Karger AG, Basel
\end{tabular} and Biochemistry Published online: May 21, 2015 www.karger.com/cpb

Table 1. Baseline characteristics of Type II DM and non-diabetic control patients. Data are shown as the mean \pm SD. * Significant differences between the two groups of individuals

\begin{tabular}{lcc}
\hline & $\begin{array}{c}\text { Diabetes } \\
(\mathrm{n}=20)\end{array}$ & $\begin{array}{c}\text { Control } \\
(\mathrm{n}=20)\end{array}$ \\
\hline Age, years & $64 \pm 9$ & $60 \pm 10$ \\
Sex, females/males & $9 / 11$ & $10 / 10$ \\
BMI, kg/m 2 & $24.7 \pm 5.1$ & $24.7 \pm 1.8$ \\
High blood pressure (BP), \% & 50 & 35 \\
Fasting plasma glucose (FPG) (mmol/L) & $8.08 \pm 0.65$ & $4.95 \pm 0.76$ \\
Hyperlipidemia, \% & 50 & 25 \\
Coronary heart disease, \% & 40 & 20 \\
\hline
\end{tabular}

isolated from peripheral blood-derived mononuclear cells using CD133-coupled magnetic microbeads (Miltenyi Biotech, USA) according to the manufacturer's instructions. CD133 ${ }^{+}$cells were resuspended in DMEM/F12 Medium (Gibco, USA) with 2\% fetal bovine serum (FBS), $10 \mu \mathrm{g} / \mathrm{L}$ vascular endothelial growth factor (VEGF), $10 \mu \mathrm{g} / \mathrm{L}$ basic fibroblast growth factor, $10 \mu \mathrm{g} / \mathrm{L}$ epidermal growth factor and $2 \mu \mathrm{g} / \mathrm{L}$ insulinlike growth factor (Peprotech, USA). After 3 days in culture at $37{ }^{\circ} \mathrm{C}$ in a $5 \% \mathrm{CO}_{2}$ incubator, non-adherent cells were removed by washing with phosphate-buffered saline (PBS), new medium was applied and the culture was maintained until day 7.

\section{Identification of EPCS}

Patent derived EPCs cultured for 7 days were incubated with fuorescein isothiocyanate conjugated lectin from Ulex europeus agglutinin 1 (FITC-UEA-1; Sigma Deisenhofen, Germany) and 1,19-dioctadecyl3,3,3939-tetramethylindocar-bocyanine perchlo-rate (Dil)-labeled acetylated low density lipoprotein (DilAc-LDL) (Molecular Probes, Life Technology, USA). Incorporation of Dil-ac-LDL and binding of FITC-UEA-1 were detected with a confocal microscope (Leica Microsystems GmbH, Germany). Dual-stained cells positive for both LDL-ac-DiI and FITC-UEA-1 were identified as EPCs [15]. The purity of the EPCs was analyzed by flow cytometry (FCM) after staining with PE-anti-CD34 and PE-anti-KDR (1:100 Santa Cruz, USA). EPCs cultured for 7 days were used for subsequent experiments.

\section{EPC proliferation assay}

Normal EPCs were cultured in plates with various concentrations of glucose or mannitol (as osmotic control). The proliferation of EPCs was determined by 3-(4, 5-dimethylthiazol-2-yl)-2, 5-diphenyltetrazolium bromide (MTT) assay. After 4, 8, 12 and 16 days of culture, EPCs were incubated with MTT $(0.5 \mathrm{mg} / \mathrm{mL}$, Sigma) for $4 \mathrm{~h}$. The blue formazen precipitate was dissolved with dimethyl sulfoxide and absorbance was measured at $550 / 650 \mathrm{~nm}$.

\section{Lentiviral Constructs, Packaging, and Transduction}

To obtain stable expression of miR-130a and MAP3K12 in EPCs, human genomic DNA was used to amplify PCR products with the following primers: miR-130a Forward, 5'-AAA CCG GTC CGC ATC AAG CCC GAA GTA T-3', and reverse, 5'-AAA GAAT TCG AGG CAG TGT CTA TCA CAA G-3'; MAP3K12 forward, TAG ACT GCA CTT TTC TGT TCC GTT TAC TCT GTT TAC ACA TTT TGC ACT TGG GAG G-3'; and reverse, GTG TAA ACA GAG TAA ACG GAA CAG AAA AGT GCA GTC TAA GTG GTT TTC TCT CCT G-3'. The miR-130a PCR products were then inserted into the PLKO.1-puro vector (Sigma-Aldrich) between the AgeI and EcoRI sites, and the MAP3K12 PCR products were cloned into the plasmid pCDH-EF1-MCS-T2A-puro (System Biosciences, SBI, CD520A-1, USA) between the XbaI and BamHI sites. These constructs were validated by sequencing. For lentivirus production, the two plasmids were individually transfected into 293T cells using psPAX2, a pMD2G packaging construct, and Lipofectamine plus reagent (Invitrogen, USA) according to the manufacturer's protocol. The medium was refreshed after $6 \mathrm{~h}$, and viral supernatant was collected after 48 h. EPCs were seeded into 6-well plates $\left(5 \times 10^{5}\right.$ cells per well) and transfected with different lentiviral vectors at an MOI of 10 as previously described [16]. After 2 days of infection, cells were selected and cloned by culture in the presence of puromycin $(2 \mu \mathrm{g} / \mathrm{mL})$ for 1 week.

\section{Luciferase reporter assay}

The forward and reverse oligonucleotides of MAP3K12-Wt were annealed to form a segment containing one putative miR-130a targeting site (UUGCACU) on the MAP3K12 3'-UTR, and amplified by PCR flanked by the SacI and PmeI sites. Similarly, a MAP3K12-Mut construct with a mutated targeting site 


\section{Cellular Physiology Cell Physiol Biochem 2015;36:712-726 \begin{tabular}{c|c}
\hline DOI: 10.1159/000430132 & (C) 2015 S. Karger AG, Basel
\end{tabular} \begin{tabular}{l|l} 
and Biochemistry Published online: May 21, 2015 & www.karger.com/cpb \\
\hline
\end{tabular} \\ Ye et al.: MiR-130a Targeting MAP3K12 Regulates EPC Function}

(UGCGAGC) was generated. The segments were cloned in the downstream of the pMIR-REPORT luciferase reporter gene vector (Ambion, Life Technologies). EPCs were seeded into 96-well plates and co-transfected with $50 \mathrm{nM}$ lenti-miR-130a or lenti-miR-control and $10 \mathrm{ng}$ of MAP3K12-Wt or MAP3K12-Mut. Cells were washed and lysed with passive lysis buffer, and luciferase activity was detected using a DualGlo Luciferase Assay System (Promega, USA).

\section{Cell transfection}

EPCs were grown to 30-40\% confluency and transfected with a human MAP3K12 siRNA (sequence cat. ZPK sc-39259, Santa Cruz Biotechnology) or control siRNA (cat. sc-37007 Santa Cruz) using Lipofectamine 2000 (Invitrogen) according to the manufacturer's recommendations. To knockdown miR-130a, a miR-130a inhibitor (anti-miR-130a, Ambion, USA) and a negative control anti-miR-1 (Ambion) were transfected into EPCs. Unless otherwise indicated, $50 \mathrm{nM}$ of RNA duplex and $200 \mathrm{nM}$ of miRNA plasmid were used for each transfection.

\section{RNA extraction and real-time quantitative PCR}

Total RNA was extracted from EPCs using the Trizol reagent according to the manufacturer's instructions (Qiagen, Germany). RNA was reverse transcribed to cDNA using PrimeScript RRT Master Mix Perfect Real Time (Takara, Japanese) and amplified using a PCR kit (Qiagen) [16]. Expression levels of miRNA130a were measured using TaqMan real-time PCR and reactions were performed using a Roche Light Cycler 480 system (Roche Diagnostics GmbH, Mannheim, Germany). U6 RNA was used as an endogenous control.

\section{Western blot analysis}

EPCs were obtained and treated with $300 \mu \mathrm{L}$ lysis buffer containing $1 \mathrm{mM}$ phenylmethanesulfonyl fluoride (Sigma) and $1 \times$ proteasome inhibitor (Sigma). Twenty micrograms of protein were loaded onto 10\% SDS-polyacrylamide gels and transferred onto PVDF membranes (Millipore, Germany). Western blotting was performed using primary antibodies against glyceraldehyde 3-phosphate dehydrogenase (GAPDH, 1:5000 dilution in 5\% milk, Santa Cruz), MAP3K12 (1:500, Abgent, USA), p-MKK7, p-JNK, p-cJun, Bcl-2 and cleaved caspase-3 (1:1000, Cell Signaling Technology, USA), and secondary goat anti-rabbit antibodies (1:4000, PerkinElmer, USA). Autoradiographs were scanned and analyzed semiquantitatively.

\section{Flow cytometry}

The apoptosis of EPCs was assessed using flow cytometric analysis. Apoptotic cells were labeled with anti-annexin V (eBioscience, USA) instead of propidium iodide (PI, eBioscience) and analyzed by fluorescence activated cell sorting (FACS).

\section{TUNEL Assay}

The apoptotic ability of EPCs was evaluated using terminal deoxynucleotidyl transferase-mediated dUTP nick-end labelling (TUNEL) assay according to the manufacturer's instructions (Promega). EPCs were counterstained with 4'6-diamino-phenylindole (DAPI, Sigma-Aldrich). TUNEL-positive cells were counted in twelve randomly selected at a magnification of $\times 200$, then the percentage of TUNEL-positive cells was analyzed.

\section{Colony-forming assay}

The colony-forming capacity of EPCs was determined as previously described [16]. Isolated EPCs were resuspended in endothelial basal medium (EBMTM-2, Cambrex, USA), and $5 \times 10^{6}$ EPCs were seeded onto fibronectin-coated 6-well plates. After two days, the non-adherent cells were collected and $1 \times 10^{6}$ cells were re-plated onto 24-well fibronectin-coated plates. On the fifth day, the EPC colony-forming units (CFUs) were manually counted in four random wells by two independent, blinded investigators. Only CFUs consisting of a central core of rounded cells surrounded by elongating and spindle-shaped cells were counted.

\section{Migration assay}

Migration assays were performed in a modified Boyden chamber (NeuroProbe). EPCs were resuspended in serum-free EBM medium and $4 \times 10^{4}$ cells were loaded into the upper chambers. The lower chamber was filled with EBM medium and VEGF (50 ng/mL). The chamber was incubated at $37{ }^{\circ} \mathrm{C}$ for $24 \mathrm{~h}$. 


\section{Cellular Physiology Cell Physiol Biochem 2015;36:712-726 \begin{tabular}{l|l|}
\hline DOI: 10.1159/000430132 & O 2015 S. Karger AG, Basel
\end{tabular} and Biochemistry Published online: May 21, 2015 www.karger.com/cpb

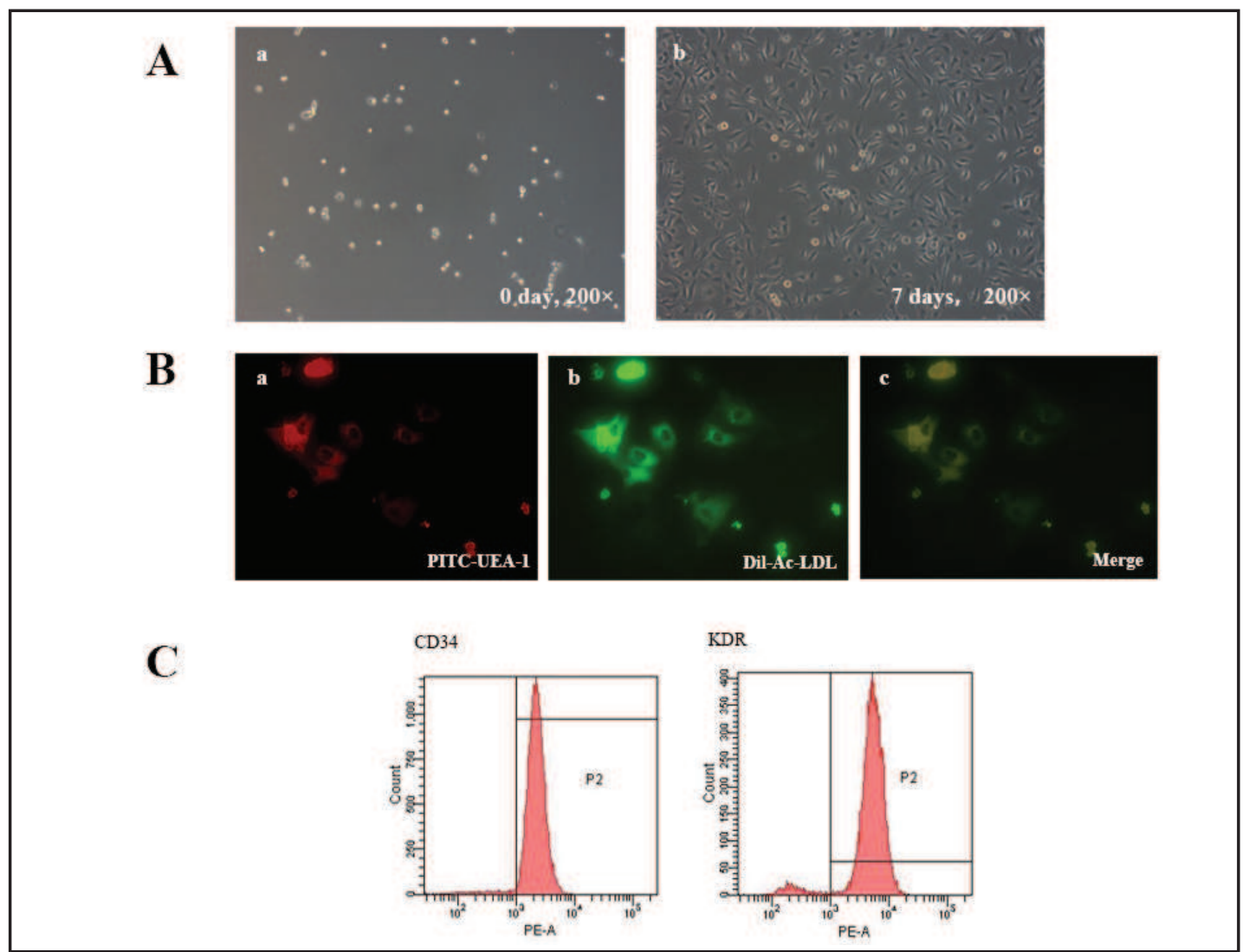

Fig. 1. A) Early EPCs were initially round and small (a), after 7 days of culture the EPCs began to develop an elongated spindle-shaped morphology (b). B) Staining of FITC-UEA-1 (a), Dil-Ac-LDL (b), and dual staining of FITC-UEA-1 and Dil-Ac-LDL (c) were detected using a confocal microscope. C) FACS analysis showed that the isolated EPCs were both positive for CD34 and KDR.

Then the lower side of the filter was washed with PBS and fixed with 4\% paraformaldeyde. The cells were stained with hematoxylin and counted in three random high-power fields $(\times 200)$ in each well.

\section{Statistical analysis}

Data from five independent experiments were expressed as mean \pm SEM and processed using SPSS 16.0 statistical software. Differences among groups were analyzed using Student's $t$-tests, and two-way ANOVA was used to compare differences among multiple groups when appropriate. A $p$ value of $<0.05$ was considered to be statistically significant.

\section{Results}

Characterization of EPCs and expression of miR-130a in diabetic EPCs

EPCs isolated by CD133-coupled magnetic micro-beads were initially round and small (Fig. 1A), and formed cell clusters after 4 days in culture. After 7 days, spindle-shaped cell appeared at the edges of the cell cluster (Fig. 1A); cells gradually became bigger and began to acquire a cobblestone-like structure on day $10.0 \mathrm{~m}$ day 8 , adherent cells were identified by co-staining with FITC-UEA-1 and Dil-ac-LDL, and double-positive cells were identified by confocal scanning microscopy (Fig. 1B). FACS analysis was used to confirm that CD $133^{+}$EPCs expressed high levels of EPC markers, including CD34 and KDR (Fig. 1C). TaqMan RT-PCR showed that miR-130a expression in diabetic patients $(n=20)$ and healthy donors $(n=20)$ were $0.57 \pm 0.11$ and $1.01 \pm 0.09$, the miR-130a expression in diabetic patients showed a significant 1.78-fold decrease compared with the healthy donors $(p<0.05)$ (Fig. 2A).

\section{KARGER}




\section{Cellular Physiology Cell Physiol Biochem 2015;36:712-726 \begin{tabular}{l|l}
\hline DOI: 10.1159/000430132 & O 2015 S. Karger AG, Basel
\end{tabular} and Biochemistry Published online: May 21, $2015 \quad$ www.karger.com/cpb
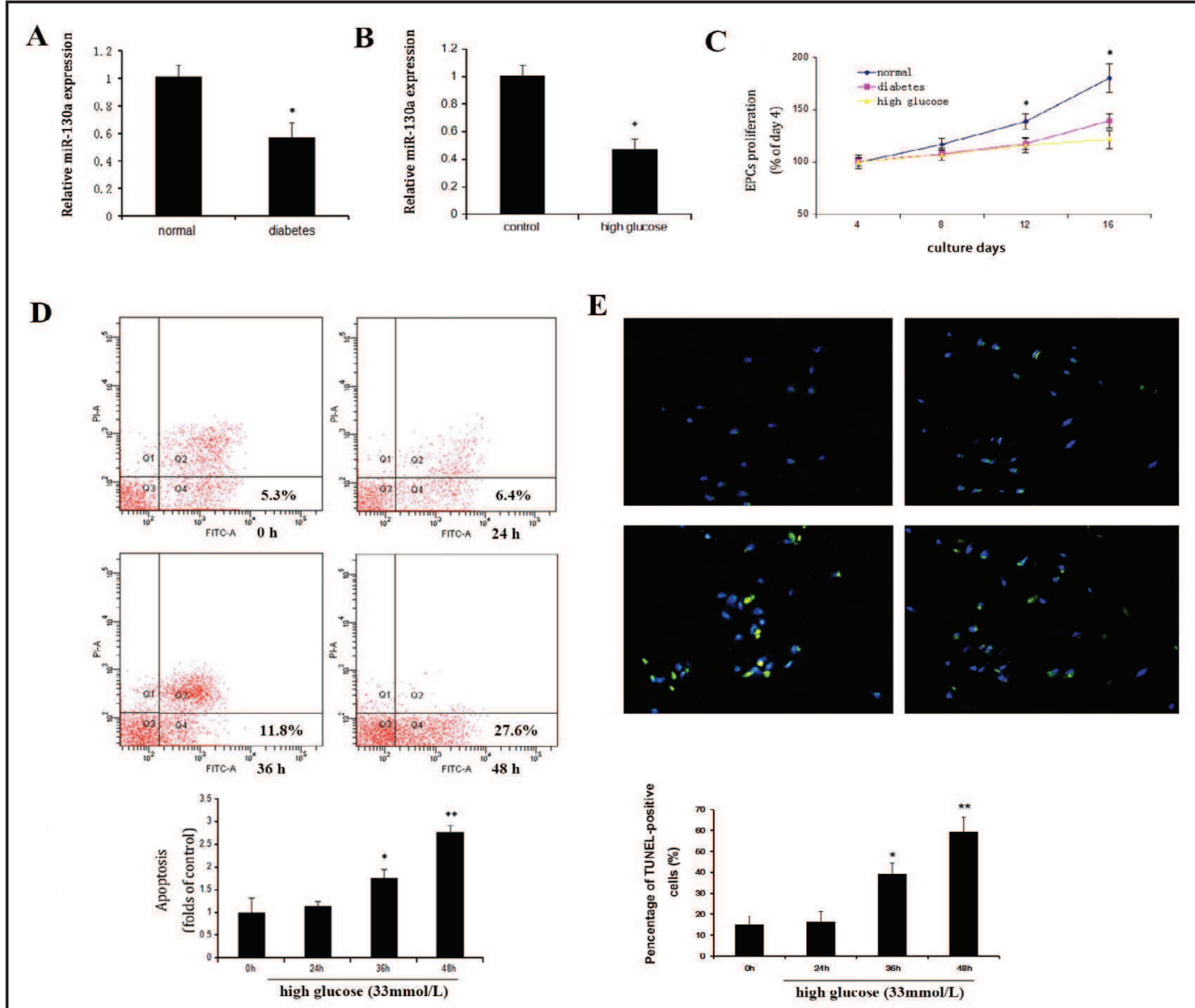

Fig. 2. A) miR-130a expression in EPCs derived from diabetes patients or healthy people were measured through RT-PCR (normalized to U6). Data are expressed as mean \pm SD ( $n=20),{ }^{*} P<0.05$ vs. normal group. B) miR-130a expression by RT-PCR (normalized to U6) in EPCs cultured under normal or high glucose (33 $\mathrm{mmol} / \mathrm{L}$ ) environment ( ${ }^{*} P<0.05$ vs. normal group). C) EPCs proliferation ability were measured in normal group, diabetes group and high glucose group, data are expressed as percentage of day $4,\left({ }^{*} P<0.05\right.$ normal group vs. diabetes group or high glucose group). D) Apoptosis of EPCs were determined at 0, 24, 36 and $48 \mathrm{~h}$ after induced by high glucose cultivation. E) Apoptosis data are expressed as folds of control group $\left({ }^{*} P<0.05\right.$ vs. $0 \mathrm{~h},{ }^{* *} P<0.05$ vs. 36 h). F) Nucleated cells are identified by blue fluorescence (DAPI DNA staining), and TUNEL-positive cells are detected by green fluorescence $(\times 200)$, apoptotic EPCs were dual stained. Similar results were obtained in five separate experiment and error bars represent the standard deviation from the mean.

\section{EPC proliferation and apoptosis under high glucose conditions}

miR-130a expression in EPCs cultured in a high glucose medium ( $33 \mathrm{mmol} / \mathrm{L}$ ) was 0.47 \pm 0.08 , while in the control group the miR-130a expression was $1.01 \pm 0.08$, it showed a significant 2.13-fold decrease of miR-130a expression under the high glucose environment $(p<0.05)$ (Fig. 2B). With the high glucose culture medium, EPC apoptosis rates were measured at different time points, they were $(5.3 \pm 0.2) \%,(6.3 \pm 0.6) \%,(12.4 \pm 1.4) \%$ and $(27.5 \pm$ $1.0) \%$ at $0 \mathrm{~h}, 24 \mathrm{~h}, 36 \mathrm{~h}$ and $48 \mathrm{~h}$ (Fig. 2D), then we calculated the folds of apoptosis rates compared with control group, they were 1.0, 1.13,1.76 and 2.76-fold increase at $0 \mathrm{~h}, 24 \mathrm{~h}, 36$ $\mathrm{h}$ and $48 \mathrm{~h}$ after high glucose culture (Fig. 2D). The cell proliferation (showed as percentage of day 4) were also evaluated in diabetic and normal EPCs, as well as normal EPCs cultured under high glucose, the cell proliferation in normal, diabetes or high glucose group were $(1.17 \pm 0.06) \%,(1.08 \pm 0.06) \%$ or $(1.07 \pm 0.05) \%$ on day $8,(1.39 \pm 0.07) \%,(1.17 \pm 0.05) \%$ or $(1.16 \pm 0.07) \%$ on day 12 and $(1.80 \pm 0.14) \%$, $(1.39 \pm 0.07) \%$ or $(1.22 \pm 0.09) \%$ on day 


\section{Cellular Physiology Cell Physiol Biochem 2015;36:712-726 \begin{tabular}{ll|l} 
and Biochemistry & Dublished online: May 21, 2015 & $\begin{array}{l}\text { C } 2015 \text { S. Karger AG, Basel } \\
\text { www.karger.com/cpb }\end{array}$ \\
\hline
\end{tabular}

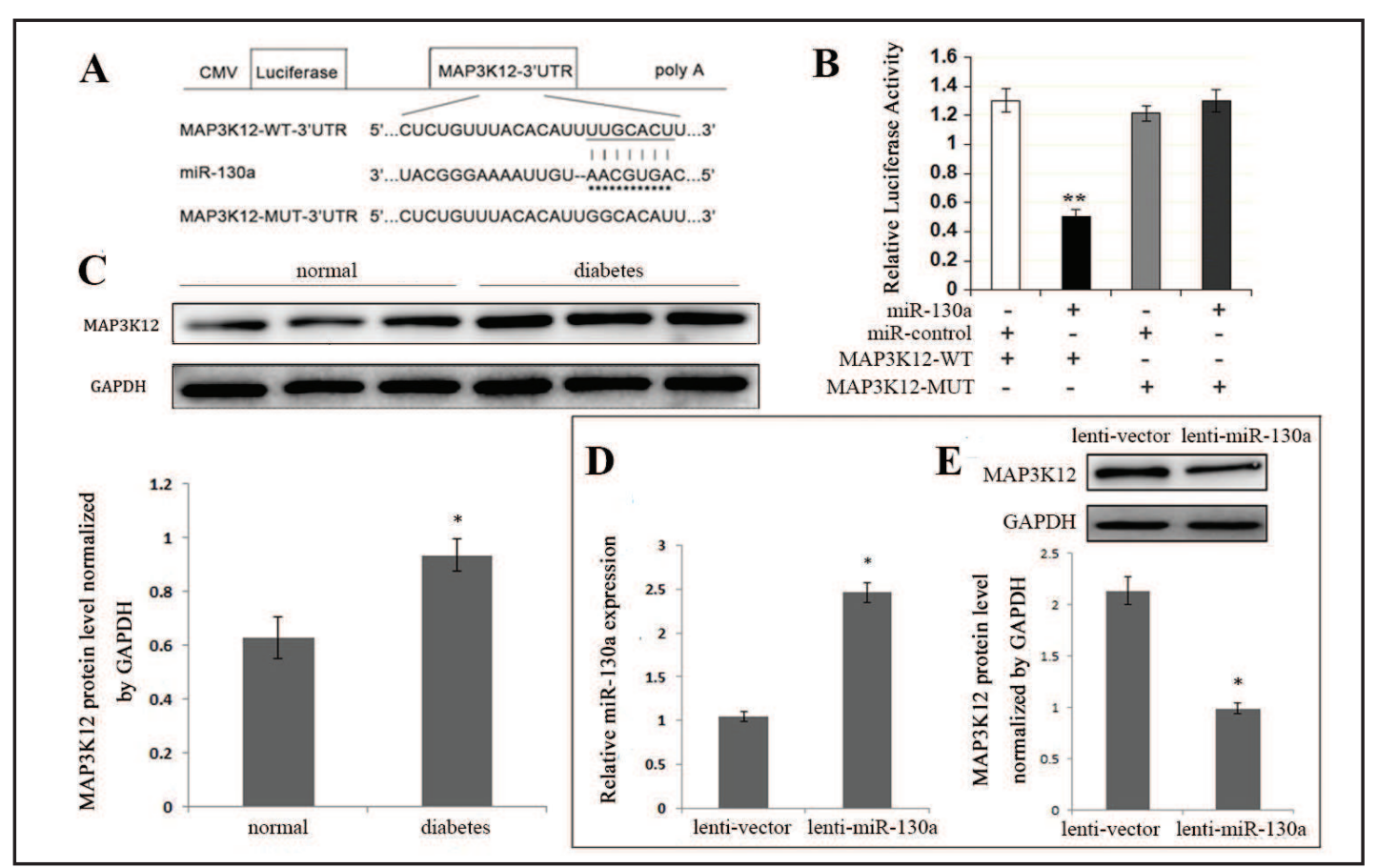

Fig. 3. A) Sequence of seed region from miR-130a and the targeting sites from 3'-UTR of MAP3K12. B) The luciferase reporter assay results were performed in the EPCs. The respective luciferase reporter plasmid pMIR-MAP3K12-3'-UTR-Wt (or -Mut) was cotransfected with either lenti-miR-130a (or lenti-miR-control) together $\left({ }^{* *} P<0.01\right)$. C) MAP3K12 protein level in EPCs derived from diabetes patients and healthy donors were measured by Western blot. D) RT-PCR analysis of miR-130a expression in EPCs transfected with lentimiR-130a $\left({ }^{*} P<0.05\right)$. E) MAP3K12 protein level was measured in EPCs transfected with lenti-miR-130a by Western blot $\left({ }^{*} P<0.05\right)$. Similar results were obtained in at least five separate experiment and error bars represent the standard deviation from the mean.

16 (Fig. 2C). Apoptosis was also examined using TUNEL assay. TUNEL-positive cells were labelled with FITC and all cells were co-stained with blue DAPI. When EPCs were exposed to high glucose, the percentage of TUNEL-positive cells, representing DNA fragmentation, were $(15.0 \pm 4.1) \%,(16.4 \pm 4.8) \%$, $(39.4 \pm 4.9) \%$ and $(59.2 \pm 6.8) \%$ at $0 \mathrm{~h}, 24 \mathrm{~h}, 36 \mathrm{~h}$ and $48 \mathrm{~h}$ respectively (Fig. 2E).

\section{MAP3K12 is a target of miR-130a in EPCs}

Computational target prediction was performed to identify relevant functional targets of miR-130a in the EPCs of diabetes patients. We hypothesized that MAP3K12, which possesses a putative miR-130a targeting site, might be a target gene of miR-130a (Fig. 3A). To confirm this, we cloned a segment of the 3'-UTR of MAP3K12 containing the miR-130a binding site into the pMIR-REPORT luciferase reporter downstream of the reporter gene. A mutant (Mut) version with a mutated binding site was also constructed. The vectors miR-130a or miRcontrol and MAP3K12-Wt or MAP3K12-Mut were co-transfected into EPCs. Overexpression of miR-130a significantly reduced the luciferase activity of MAP3K12-Wt, whereas luciferase activity was unaffected by miR-control with mutated nucleotides in the miR-130a seed binding site. Meanwhile, miR-130a-mediated repression of luciferase activity was abolished by the mutant putative binding site of the MAP3K12-Mut (Fig. 3B). Western blot analysis showed that MAP3K12 expression was higher in EPCs derived from DM patients $(0.93 \pm$ 0.06 , normalized by GAPDH) than in those from healthy donors $(0.62 \pm 0.07)(p<0.05)$ (Fig. 3C). To further verify our hypothesis, the expression levels of miR-130a were assessed in normal EPCs transfected with a lentivirus carrying miR-130a or miR-control, which showed that miR-130a expression was significantly upregulated in the miR-130a transfected group ( $p<0.05$ vs miR-control group) (Fig. 3D), demonstrating that miR-130a was successfully 


\section{Cellular Physiology Cell Physiol Biochem 2015;36:712-726

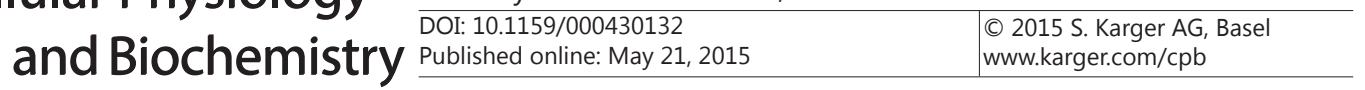

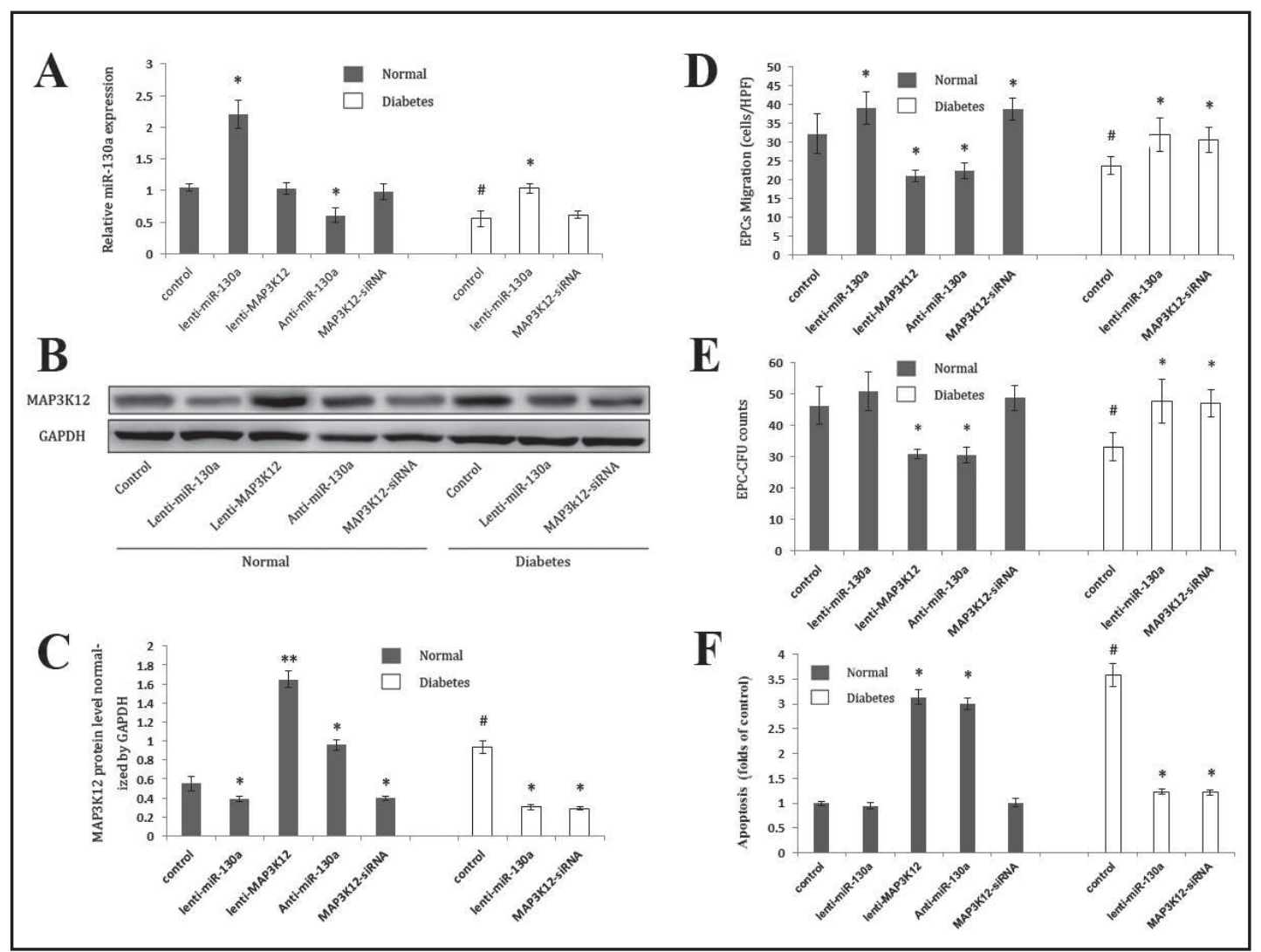

Fig. 4. Control group: cells infected with empty vector. Lenti-miR-130a group: cells transfected with lentivirus expressed miR-130a. Lenti-MAP3K12 group: cells transfected with lentivirus expressed MAP3K12 mRNA. Anti-miR-130a group: cells transfected with miR-130a inhibitor. MAP3K12-siRNA group: cells transfected with human MAP3K12 siRNA to knock down MAP3K12 mRNA. A) miR-130a expression by RT-PCR (normalized to U6). (B, C) MAP3K12 protein levels by Western blot and densitometric measurement results of MAP3K12. (D-F) Functional assays of EPCs: migration (D), colony formation (E) and apoptotic cells (F)

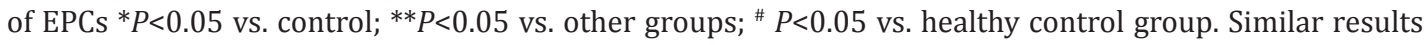
were obtained in at least five separate experiment and error bars represent the standard deviation from the mean.

overexpressed. Moreover, the enhanced miR-130a expression in EPCs significantly repressed MAP3K12 protein expression compared with the miR-control group $(P<0.05)$ (Fig. 3E).

\section{Inhibition of miR-130a and increase of MAP3K12 impair EPC function}

To investigate the role of miR-130a in EPC function via its target MAP3K12, cells were transfected as follows: 1) EPCs derived from healthy donors were transfected with (a) empty vector as control, (b) lenti-miR-130a to overexpress miR-130a, (c) lenti-MAP3K12 to overexpress the MAP3K12 protein, (d) anti-miR-130a to decrease endogenous miR130a level, or (e) MAP3K12-siRNA to decrease MAP3K12 levels; 2) EPCs derived from diabetes patients were transfected with (a) empty vector as control, (b) lenti-miR-130a for recovery of miR-130a level, (c) MAP3K12-siRNA to decrease the elevated MAP3K12 protein level, (d) anti-miR-130a to inhibit relative miR-130a expression or (e) lenti-MAP3K12 to overexpress the MAP3K12. After the different treatments, we confirmed that the miR-130a was significantly overexpressed in both lenti-miR-130a groups and decreased in the antimiR-130a group in normal EPCs to mimic the situation in diabetic EPCs $(P<0.05$ vs. control $)$ (Fig. 4A). The expression of MAP3K12 was altered depending on the different treatments as follows: in normal EPCs, MAP3K12 levels were decreased by overexpression of miR-130 or siRNA knock-down of MAP3K12, whereas they were increased by downregulation of miR- 


\section{Cellular Physiology Cell Physiol Biochem 2015;36:712-726 \begin{tabular}{ll|l} 
and Biochemistry & $\begin{array}{l}\text { DOI: 10.1159/000430132 } \\
\text { Published online: May 21, } 2015\end{array}$ & $\begin{array}{l}\text { C 2015 S. Karger AG, Basel } \\
\text { www.karger.com/cpb }\end{array}$ \\
\hline
\end{tabular}

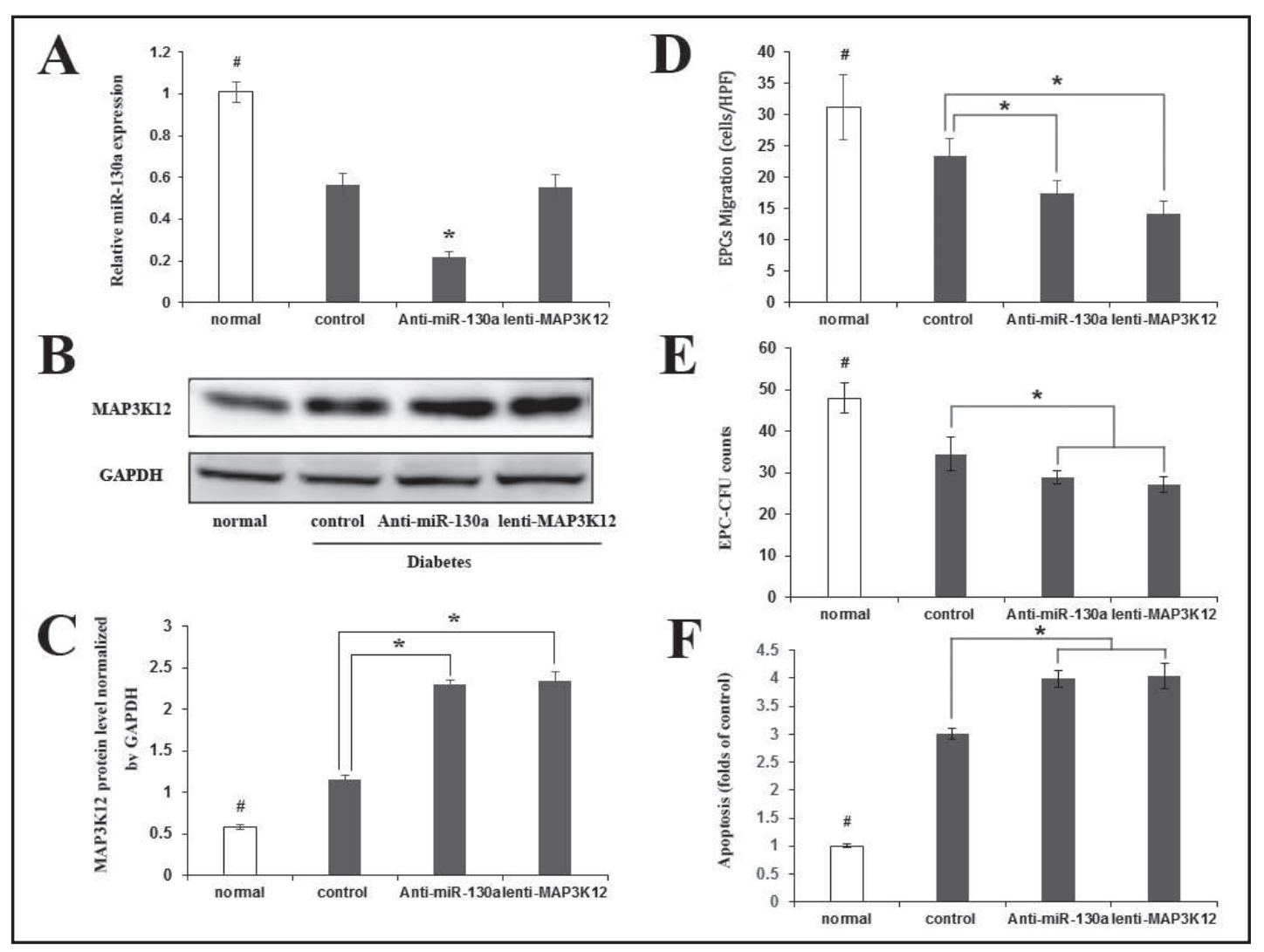

Fig. 5. Control group: cells transfected with empty vector. Anti-miR-130a group: cells transfected with miR130a inhibitor. Lenti-MAP3K12 group: cells transfected with lentivirus expressed MAP3K12 mRNA. A) miR130a expression by RT-PCR (normalized to U6). (B, C) MAP3K12 protein levels by Western blot and densitometric measurement results of MAP3K12. (D-F) Functional assays of EPCs: migration (D), colony formation (E) and apoptotic cells (F) of EPCs ${ }^{*} P<0.05$ vs. control; ${ }^{\#} P<0.05$ vs. other groups. Similar results were obtained in at least five separate experiment and error bars represent the standard deviation from the mean.

130a or overexpression of MAP3K12 mRNA ( $P<0.05$ vs. control) (Fig. 4B, 4C). Meanwhile, the overexpression of either miR-130a or MAP3K12-siRNA also similarly decreased the MAP3K12 protein level in diabetic EPCs ( $P<0.05$ vs. control) (Fig. 4B, 4C). We also overexpressed the MAP3K12 or downregulated miR-130a in diabetic EPCs to further increase the MAP3K12 protein level in diabetic EPCs ( $P<0.05$ vs. control) (Fig. 5A, 5B, 5C).

Upregulation of miR-130a or downreguation of MAP3K12 mRNA both promoted significant cell migration ability in normal and diabetic EPCs, and increased the colony formation but reduced the apoptosis in diabetic EPCs ( $P<0.05$ vs. control) (Fig. 4D, 4E, 4F). Downregulation of miR-130a or upreguation of MAP3K12 mRNA significantly reduced migration and colony formation and increased apoptosis in both normal and diabetic EPCs $(P<0.05$ vs. control) (Fig. 4D, 4E, 4F, 5D, 5E, 5F)

\section{MiRNA-130a potentially impairs EPC function through the JNK pathway}

Cell proliferation in response to mitogenic stimulation involves phosphorylated JNK. Therefore, we tested whether miR-130a affects the JNK signal pathway. Anti-miR-130a was transfected into healthy donor-derived EPCs to knock down miR-130a expression, and antimiR-1 was transfected into normal EPCs as control. Diabetic EPCs were transfected with control siRNA or MAP3K12 siRNA to knock down MAP3K12 expression. The protein levels of MAP3K12, phosphorylated MKK7, JNK and c-Jun, Bcl-2 and cleaved caspase-3 were also measured by western blotting (Fig. 6A). Normal EPCs treated with anti-miR-130a showed upregulation of the expression of MAP3K12, p-MKK7, p-JNK and p-c-Jun $(P<0.05$ vs. control) 


\section{Cellular Physiology Cell Physiol Biochem 2015;36:712-726 \begin{tabular}{l|l|l}
\hline DOI: 10.1159/000430132 & O 2015 S. Karger AG, Bas
\end{tabular} \\ Ye et al.: MiR-130a Targeting MAP3K12 Regulates EPC Function}

Fig. 6. Anti-miR-130a group: healthy donor-derived EPCs infected with anti-miR-130a; Anti-miR-1 group: healthy donor-derived EPCs infected with anti-miR-1; MAP3K12 siRNA group: diabetic patient-derived EPC infected with MAP3K12 siRNA; Control siRNA group: diabetic patient-derived EPC infected with control siRNA. A) MAP3K12, p-MKK7, p-JNK, p-c-Jun, Bcl-2 and cleaved caspase-3 protein level by western blot. (B-G) Protein levels normalized by GAPDH. ${ }^{* \#} P<0.05$. Similar results were obtained in at least five separate experiment and error bars represent the standard deviation from the mean.

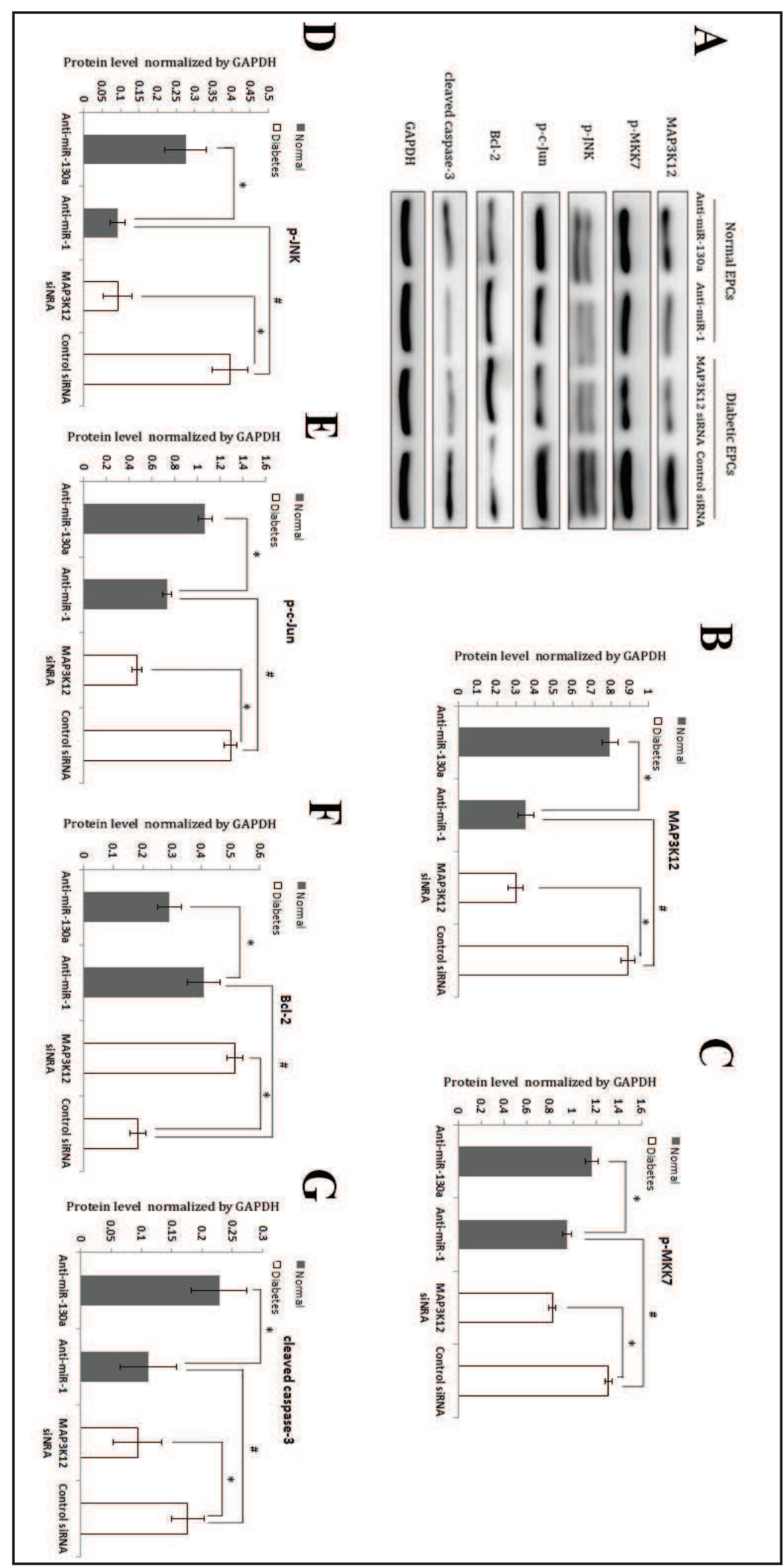

(Fig. 6B, 6C, 6D, 6E). Downregulation of MAP3K12 expression by MAP3K12 siRNA in diabetic EPCs (Fig. 5B) resulted in the down regulation of phosphorylated MKK7, JNK and c-Jun $(P<0.05$ vs. control) (Fig. 6C, 6D, 6E). In addition, in diabetic EPCs, Bcl-2 protein levels were 


\section{Cellular Physiology Cell Physiol Biochem 2015;36:712-726 \begin{tabular}{l|l} 
DOI: 10.1159/000430132 & \\
and Biochemistry Published online: May 21, 2015 & $\begin{array}{l}\text { 2015 S. Karger AG, Ba } \\
\text { www.karger.com/cpb }\end{array}$ \\
\cline { 2 - 3 } &
\end{tabular} \\ Ye et al.: MiR-130a Targeting MAP3K12 Regulates EPC Function}

Fig. 7. Anti-miR-130a group: diabetic patient-derived EPCs transfected with antimiR-130a; Anti-miR-1 group: diabetic patient-derived EPCs transfected with anti-miR-1; MAP3K12 siRNA group: healthy donor-derived EPCs transfected with MAP3K12 SiRNA; Control siRNA group: healthy donor-derived EPCs transfected with control siRNA. A) MAP3K12, p-MKK7, p-JNK, p-c-Jun, Bcl-2 and cleaved caspase- 3 protein level by western blot. (B-G) Protein levels normalized by GAPDH. ${ }^{*} P<0.05$. Similar results were obtained in at least five separate experiment and error bars represent the standard deviation from the mean.

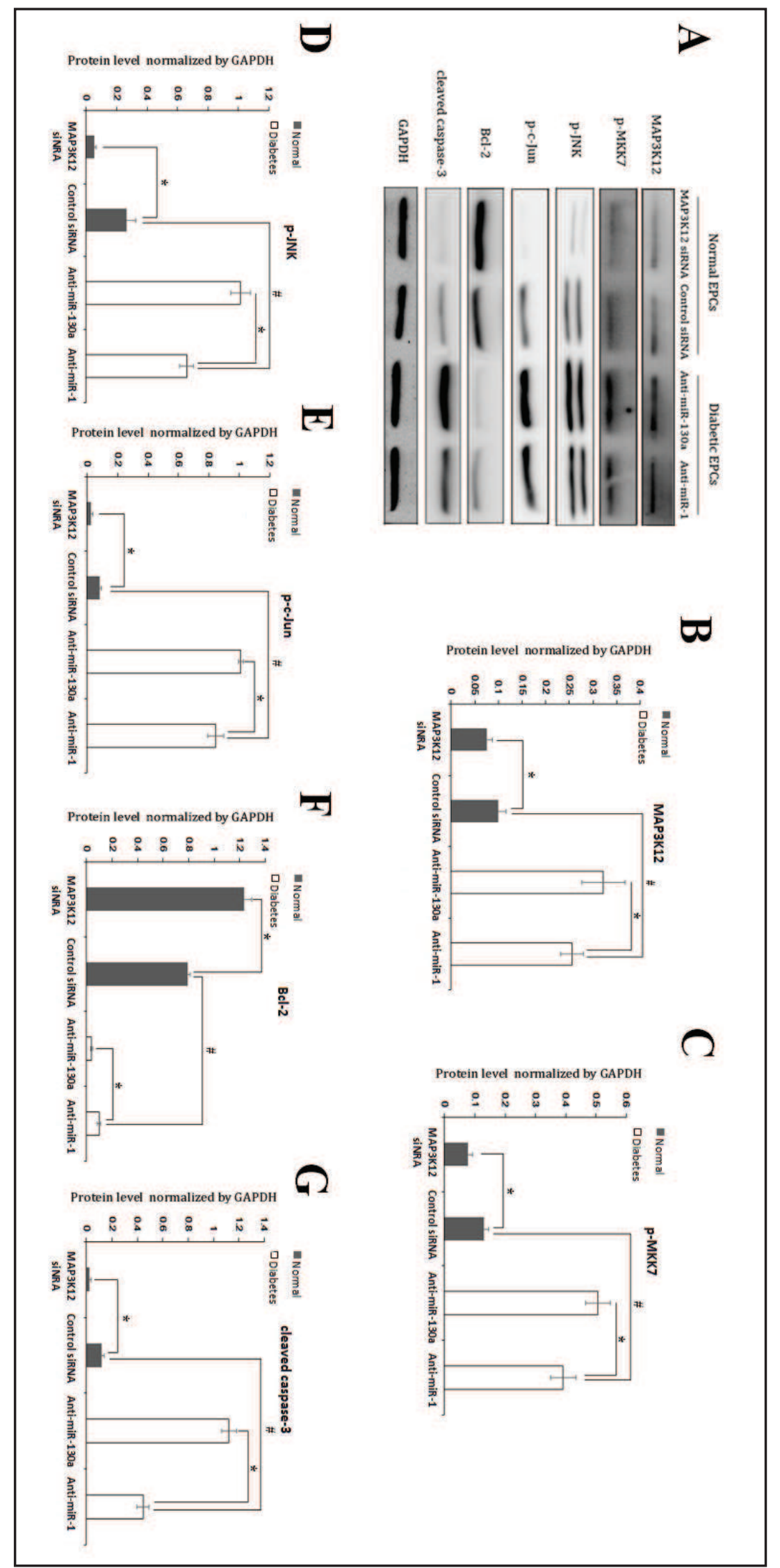




\section{Cellular Physiology Cell Physiol Biochem 2015;36:712-726

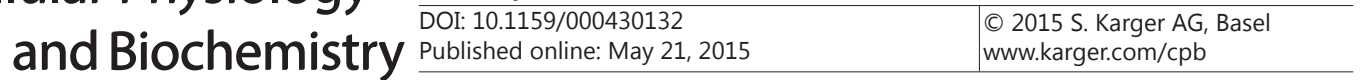

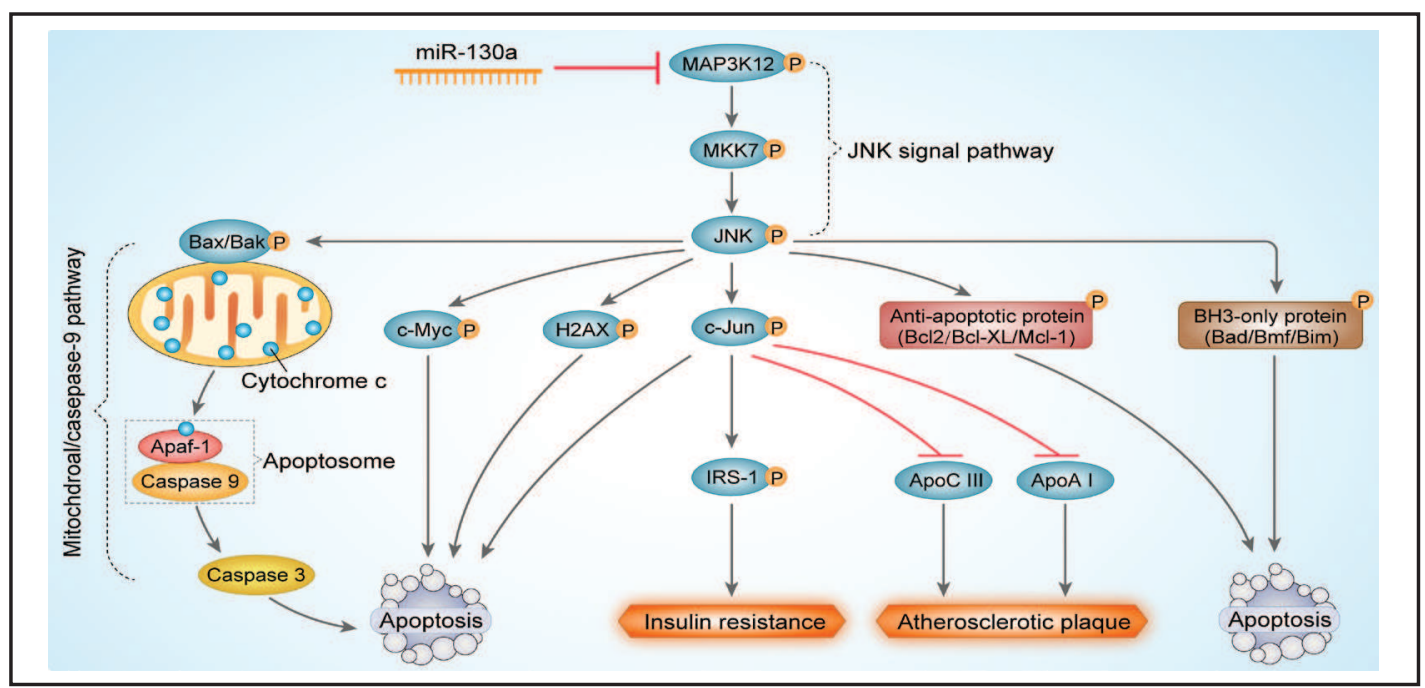

Fig. 8. MiR-130a targeting MAP3K12 negatively regulates JNK signal transduction pathways.

lower than those in normal EPCs, and the level of cleaved caspase- 3 was higher $(P<0.05)$ (Fig. 6F, 6G). However, down regulation of miR-130a in normal EPCs decreased Bcl-2 level and increased the cleavage of caspase-3, whereas down regulation of MAP3K12 in diabetic EPCs had the opposite effect ( $P<0.05$ vs. control) (Fig. 6F, 6G).

On the other hand, we also transfected anti-miR-130a into diabetic EPCs and MAP3K12 siRNA into normal EPC, and measured the JNK signal pathway downstream protein levels or apoptosis related protein levels (Fig. 7A). Normal EPCs treated with MAP3K12 siRNA showed downregulation of the expression of MAP3K12, p-MKK7, p-JNK and p-c-Jun $(P<0.05$ vs. control) (Fig. 7B, 7C, 7D, 7E). Downregulation of miR-130a by anti-miR-130a in diabetic EPCs resulted in the upregulation of MAP3K12 and phosphorylated MKK7, JNK and c-Jun $(P<0.05$ vs. control) (Fig. 7B, 7C, 7D, 7E). Meanwhile, downregulation of MAP3K12 in normal EPCs further increased Bcl-2 level and decreased the cleavage of caspase-3, whereas downregulation of miR-130a in diabetic EPCs further decreased the Bcl-2 but promote more cleavage of caspase-3 ( $P<0.05$ vs. control) (Fig. 7F, 7G).

\section{Discussion}

One of the complications of DM is vessel endothelial dysfunction, and the dysfunction of EPCs may promote the progression of diabetic complications. DM is characterized by a two to four-fold increased risk of cardiovascular diseases, and patients with pre-diabetic conditions, such as impaired fasting glucose and impaired glucose tolerance, are at increased risk of cardiovascular disorders [17]. Endothelial dysfunction is thought to be the first step of the adverse sequence of events that leads to the atherosclerotic process $[18,19]$. This is generally attributed to the adverse effects of hyperglycemia and oxidative stress on vascular biology. High glucose induces apoptosis through the activation of stress-activatedMAP kinase (SAPK)/JNK (but not the other MAPK subfamilies ERK 1/2 and p38) in human EPCs [5]. However, the mechanism regulating its upstream activation under high glucose conditions remains unclear.

MiRNA dysregulation has been observed in various pathological processes, including the EPC-mediated pathogenesis of diabetic complications. We screened miRNAs showing alterations in EPCs derived from type II DM patients and found that miR-130a was significantly down regulated, which is consistent with previous findings [18], and suggests that the low level of miR-130a in diabetic EPCs may be involved in the functional impairment of EPCs in these patients.

MiR-130a appears to be widely expressed in diverse cell types and it regulates various cell functions by targeting different proteins $[20,21]$. To verify the influence of high glucose 


\section{Cellular Physiology Cell Physiol Biochem 2015;36:712-726

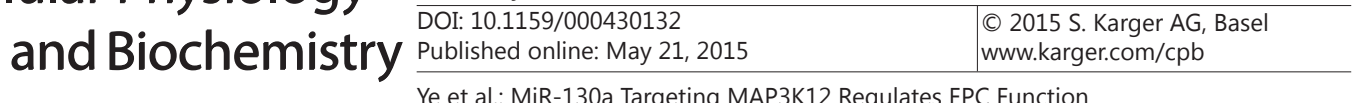

on the expression of miR-130a, normal EPCs were cultured under high glucose conditions (33 mmol/L) to mimic the diabetic microenvironment. It was shown that EPC proliferation rate was significantly higher in normal group than the diabetes or high glucose group. We also found that miR-130a was downregulated under high glucose and induced cell apoptosis. These results suggested a possible relationship between the downregulation of miRNA-130a and EPC apoptosis.

We searched for targets of miR-130a using several databases and identified MAP3K12 as a potential target of miR-130a in EPCs. MAP3K12 phosphorylates and activates the MAPKK isoforms MKK4 and MKK7, which in turn phosphorylate and activate JNK [13]. Phosphorylation of c-Jun by JNK increases transcriptional activity by activating activator protein-1 (AP-1), linked to JNK-dependent apoptosis [22]. We therefore addressed a potential molecular mechanism underlying miR-130a-mediated effects by focusing on the role of MAP3K12 in endothelial injury of diabetes (Fig. 6). We hypothesized that the reason for the decreased proliferation ability of diabetic EPCs could be high-glucose-induced miR-130a downregulation. This could exert an opposite positive effect on the JNK signaling pathway because of the decreased targeting of MAP3K12 by miR-130a, resulting in increased JNKdependent apoptosis of EPCs and impaired EPC function.

To verify our hypothesis, we performed a luciferase reporter assay to demonstrate that miR-130a binds directly to specific complementary sites in the 3'-UTR of MAP3K12, and overexpression of miR-130a in EPCs dramatically decreased the mRNA and protein expression levels of MAP3K12. Functional assays showed that diabetic EPCs had impaired function compared to normal EPCs, which appeared to be partly associated with the downregulation of miR-130a and the effect on its targets MAP3K12. Inhibition of miR-130a in normal EPCs adversely affected EPC function, as anti-miR-130a significantly reduced migration and colony formation and increased apoptosis in EPCs, compared to the negative control. These results suggested that miR-130a is fundamental for the maintenance of normal EPC function by targeting MAP3K12.

To verify the results of the gain-of-function study, we further explored whether the involvement of miR-130a in EPC function was mediated by the regulation of the expression level of MAP3K12 in diabetic EPCs. In cells transfected with either lenti-miR-130a or MAP3K12-siRNA, MAP3K12 was down regulated at the mRNA and protein levels; however, the colony formation and migration capacity of EPCs was increased and apoptosis was decreased. These initial experiments suggested that miR-130a may negatively regulate the expression of MAP3K12 by directly targeting the $3^{\prime}$ UTR of its mRNA, thus affecting the viability of EPCs.

To determine whether miR-130a inhibits the JNK pathway to exert an anti-apoptosis effect, lenti-miR-130a or anti-miR-130a was transfected into EPCs to regulate the level of MAP3K12. The results showed that the protein levels of phosphorylated MKK7, JNK and c-Jun were modulated by the up or down regulation of MAP3K12 expression, indicating that miR-130a may regulate the downstream kinases cascade of the JNK signaling pathway by targeting MAP3K12. In addition, we found that activation of JNK kinases can decrease Bcl-2 expression and increase the levels of cleaved-caspase-3. Several studies have shown that the anti-apoptotic proteins $\mathrm{Bcl}-2$ and $\mathrm{Bcl}-\mathrm{X}_{\mathrm{L}}$ are phosphorylated by JNK and their anti-apoptotic function is inhibited [23-25]. Bcl-2 proteins are known to regulate the release of mitochondrial cytochrome $\mathrm{c}$ and other mitochondrial pro-apoptotic molecules, which converge on the activation of the effector caspase-3 [26]. The other potential targets of JNK in pro-apoptotic signaling in mitochondria include the pro-apoptotic $\mathrm{BH}_{3}$-only members (Bad, Bmf, Bim), which can be phosphorylated by JNK and cause increased apoptosis in cells exposed to UV radiation [26-28]. It has been also reported that the pro-apoptotic protein Bax/Bak may be inactivated by JNK-mediated phosphorylation [29]. Meanwhile, H2AX, a histone H2A variant, was shown to contribute to apoptosis by JNK-mediated phosphorylation [30, 31]. However, early transient JNK activation promotes cell survival, whereas prolonged JNK activation can mediate apoptosis [32]. In the present study, prolonged downregulation of miR-130a under high glucose conditions in diabetic EPCs might result in sustained JNK activation, with the 


\section{Cellular Physiology Cell Physiol Biochem 2015;36:712-726

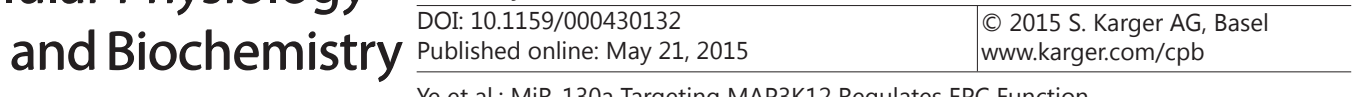

consequent increase in the apoptosis rate. In diabetic EPCs transfected with miR-130a, which showed stable recovery of miR-130a, downregulation of MAP3K12 negatively regulated the JNK signal pathway and the apoptosis rate in this type of EPCs was markedly decreased. In normal EPCs transfected lenti-MAP3K12, which showed stable overexpressed MAP3K12 and sustained activation of the JNK signal pathway, the apoptosis rate became higher. These results significantly contribute to our understanding of the critical role of miR-130a in the regulation of JNK activation mediated by its targeting of MAP3K12.

MAP3K12 activation of the JNK pathway and its role in the impaired regenerative response of primary sensory neurons has been reported $[11,33]$, however, its role in impaired EPCs has not been reported to date. Our initial experiments showed that miR130a may negatively regulate the expression of MAP3K12 and affect the EPC function such as migration, proliferation and colony formation ability. Biochemical studies established that JNK phosphorylates insulin receptor substrate-1 (IRS-1) to partly contribute to insulin resistance and type 2 diabetes $[34,35]$. The c-Jun transcription factor represses apolipoprotein CIII (Apo CIII) promoter activity, and JNK represses apolipoprotein A-I (ApoAI) promoter activity, thus reducing the number of atherosclerotic plaques [36]. Therefore, JNK represents an exciting new drug target for DM and its vascular complications because of the diverse number of mechanisms involved (shown as Fig. 8) [23].

In summary, the present study demonstrated that miR-130a is dysregulated in the EPCs of patients with DM. We showed that miR-130a plays an important role in impairing EPC function by directly targeting MAP3K12, a newly identified target gene. These results suggest that downregulation of miR-130a and activation of the JNK signaling pathway may provide targets for strategic therapeutic applications in diabetes mellitus.

\section{Disclosure Statement}

There are no competing interests.

\section{References}

1 Hill JM, Zalos G, Halcox JP, Schenke WH, Waclawiw MA, Quyyumi AA, Finkel T: Circulating endothelial progenitor cells, vascular function, and cardiovascular risk. N Engl J Med 2003;348:593-600.

2 Werner N, Kosiol S, Schiegl T, Ahlers P, Walenta K, Link A, Bohm M, Nickenig G: Circulating endothelial progenitor cells and cardiovascular outcomes. N Engl J Med 2005;353:999-1007.

3 van den Oever IA, Raterman HG, Nurmohamed MT, Simsek S: Endothelial dysfunction, inflammation, and apoptosis in diabetes mellitus. Mediators Inflamm 2010;2010:792393.

4 Moreno PR, Fuster V: New aspects in the pathogenesis of diabetic atherothrombosis. J Am Coll Cardiol 2004; 44:2293-2300.

5 Ho FM, Lin WW, Chen BC, Chao CM, Yang CR, Lin LY, Lai CC, Liu SH, Liau CS: High glucose-induced apoptosis in human vascular endothelial cells is mediated through NF-kappaB and c-Jun NH2-terminal kinase pathway and prevented by PI3K/Akt/eNOS pathway. Cell Signal 2006;18:391-399.

6 Valencia-Sanchez MA, Liu J, Hannon GJ, Parker R: Control of translation and mRNA degradation by miRNAs and siRNAs. Genes Dev 2006;20:515-524.

7 Meng S, Cao JT, Zhang B, Zhou Q, Shen CX, Wang CQ: Downregulation of microRNA-126 in endothelial progenitor cells from diabetes patients, impairs their functional properties, via target gene Spred-1. J Mol Cell Cardiol 2012;53:64-72.

8 Meng S, Cao J, Wang L, Zhou Q Li Y, Shen C, Zhang X, Wang C: MicroRNA 107 partly inhibits endothelial progenitor cells differentiation via HIF-1beta. PLoS One 2012;7:e40323.

9 Zhao T, Li J, Chen AF: MicroRNA-34a induces endothelial progenitor cell senescence and impedes its angiogenesis via suppressing silent information regulator 1. Am J Physiol Endocrinol Metab 2010;299:E110-116.

10 Meng S, Cao J, Zhang X, Fan Y, Fang L, Wang C, Lv Z, Fu D, Li Y: Downregulation of microRNA-130a contributes to endothelial progenitor cell dysfunction in diabetic patients via its target Runx3. PLoS One 2013;8:e68611.

11 Itoh A, Horiuchi M, Bannerman P, Pleasure D, Itoh T: Impaired regenerative response of primary sensory neurons in ZPK/DLK gene-trap mice. Biochem Biophys Res Commun 2009;383:258-262. 


\section{Cellular Physiology Cell Physiol Biochem 2015;36:712-726 \begin{tabular}{c|l} 
DOI: 10.1159/000430132 & (c) 2015 S. Karger AG, Basel
\end{tabular} \begin{tabular}{l|l} 
and Biochemistry Published online: May 21, 2015 & www.karger.com/cpb \\
\hline
\end{tabular} \\ Ye et al.: MiR-130a Targeting MAP3K12 Regulates EPC Function} apoptosis. J Cell Biol 2013;202:747-763.

34 Aguirre V, Uchida T, Yenush L, Davis R, White MF: The c-Jun NH(2)-terminal kinase promotes insulin resistance during association with insulin receptor substrate-1 and phosphorylation of Ser(307). J Biol Chem 2000;275:9047-9054.

35 Lee YH, Giraud J, Davis RJ, White MF: c-Jun N-terminal kinase (JNK) mediates feedback inhibition of the insulin signaling cascade. J Biol Chem 2003;278:2896-2902.

36 Ricci R, Sumara G, Sumara I, Rozenberg I, Kurrer M, Akhmedov A, Hersberger M, Eriksson U, Eberli FR, Becher B, Boren J, Chen M, Cybulsky MI, Moore KJ, Freeman MW, Wagner EF, Matter CM, Luscher TF: Requirement of JNK2 for scavenger receptor A-mediated foam cell formation in atherogenesis. Science 2004;306:1558-1561. 